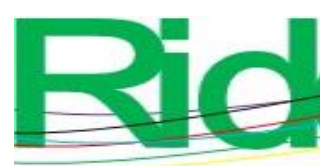

Revista Iberoamericana para la Investigación y el Desarrollo Educativo ISSN $2007-7467$

https://doi.org/10.23913/ride.v11i22.848

Artículos científicos

\title{
Sistemas de tutoría inteligente y su aplicación en la educación superior
}

\section{Intelligent Tutoring Systems and their application in higher education}

\section{Sistemas de tutoria inteligentes e sua aplicação no ensino superior}

\author{
Mario Humberto Rodríguez Chávez \\ Universidad Politécnica de Victoria, México \\ mrodriguezc@upv.edu.mx \\ https://orcid.org/0000-0002-5421-427X
}

\section{Resumen}

El sector educativo está cambiando con la evolución e implantación de nuevas tecnologías. Hasta hace poco, las plataformas virtuales para el desarrollo de actividades educativas, el software colaborativo de e-learning e incluso los dispositivos móviles como las tabletas electrónicas eran recursos impensables en un aula. Sin embargo, debido a la evolución de la tecnología, se han venido desarrollando nuevas herramientas didácticas para fortalecer la educación, entre estas tenemos sistemas tutores inteligentes (ITS), los cuales están diseñados para fortalecer el aprendizaje dentro y fuera del aula. Por eso, el objetivo principal de este trabajo es el análisis del uso y características de los sistemas de tutoría inteligente como programas de enseñanza asistida por computadora, los cuales utilizan técnicas de inteligencia artificial para la representación del conocimiento y así proponer/dirigir una enseñanza preparada para comportase como un experto capaz de realizar un diagnóstico situacional del alumno y, en relación con ello, ofrecer una solución o acción. Para cumplir este objetivo se estudiaron los diferentes componentes de un sistema de tutoría inteligente y su implementación en diferentes áreas de conocimiento de las ciencias.

Palabras clave: educación, inteligencia artificial, programación, tutor inteligente. 


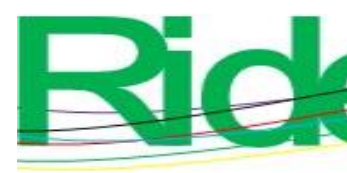

Revista Iberoamericana para la Investigación y el Desarrollo Educativo ISSN $2007-7467$

Wolf (1984) define los ITS como sistemas que modelan la enseñanza, el aprendizaje, la comunicación y el dominio del conocimiento del especialista y el entendimiento del estudiante sobre ese dominio. Asi mismo Giraffa, Nunes y Viccari (1997) en su investigación los delimitan los ITS como sistemas que incorpora técnicas de IA (inteligencia artificial) a fin de crear un ambiente que considere los diversos estilos cognitivos de los alumnos que utilizan el programa. Entre los ITS desarrollados siguiendo las ideas de Carbonell (1970) y con base en paradigmas de programación convencional (no a través del paradigma de agentes inteligentes) se pueden destacar los siguientes: Scholar (Carbonell, 1970), Why (Stevens y Collins, 1977), Sophie (Brown et al., 1989), Guidon (Clancey et al., 1991), West (Burton et al., 1981), Buggy (Brown y Burton, 1978), Debuggy (Brown et al., 1989), Steamer (Stevens y Collins, 1977), Meno (Wolf, 1984), Proust (Johnson, 1986), Sierra (VanLehn, 1988). Luego surgen Andes (Gertner, Conati y VanLehn, 1998; Gertner y Van Lehn et al., 2000) en el Pittsburgh Science of Learning Center's LearnLab, que su consorcio con miembros de Carnegie Mellon University, University of Pittsburgh y Carnegie Learning. Metutor es un tutor de medios-fines del Department of Computer Science, U. S. Naval Postgraduate School, Monterey (Galvin, 1994; Rowe, 1998).

Un estudiante de nuevo ingreso que toma un primer curso en algoritmos y programación computacional desconoce varias temáticas relacionadas con entender un texto, analizar y resolver un problema matemático o plantear una estrategia de solución para llevar a cabo la implementación de un algoritmo a una problemática presentada. El éxito de superar estos obstáculos está relacionado con dos elementos principales en el proceso de enseñanzaaprendizaje: el estudiante y el profesor. El primero al tener tenacidad e iniciativa de aprender, entendiendo el valor que tiene el aprendizaje para el éxito de emplearse como experto en la industria del software y el segundo al identificar las fortalezas y debilidades del alumno para plantearle un plan de acción que permita la autorrealización del alumno, y que aumente la tolerancia a la frustración de este.

El elemento clave en la dinámica antes mencionada es saber cuál es la usabilidad de los ITS en la educación. Para responder la pregunta de investigación es necesario identificar las fortalezas y debilidades del alumno, así como la necesidad del refuerzo de temas relacionados con la programación de computadoras y solución de problema. De esta manera, los sistemas de tutoría inteligente ayudan a los estudiantes en el aula y fuera de ella a aprender diferentes temas, pues el ITS hace la función del profesor para ofrecer una alternativa de estudio y aprendizaje de los diferentes tópicos. 


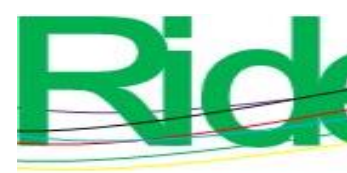

Revista Iberoamericana para la Investigación y el Desarrollo Educativo ISSN $2007-7467$

En definitiva, la investigación plantea analizar los diferentes ITS utilizados a nivel superior para el apoyo en aprendizaje de diferentes áreas educativas, así como las técnicas de IA utilizadas para su desarrollo e implementación.

\section{Marco conceptual}

Rodríguez (2019) plantea que en la actualidad los sistemas tradicionales de aprendizaje a través de la computadora necesitan mejoras, las cuales son más evidentes con el incremento de la exposición del Internet y el crecimiento de sistemas conocidos como educación virtual. Ovalle y Jiménez (2006) detallan que la dificultad de suministrar una enseñanza individualizada adaptada a las necesidades y características específicas del alumno exige un cambio de paradigma en el proceso de aprendizaje a través de computadora.

Dentro de este marco, Rodríguez (2019) explica que existen diversos sistemas educativos computarizados que utilizan técnicas de la inteligencia artificial (IA), los cuales — de acuerdo con Ovalle y Jiménez (2006) — buscan aplicarlas a un desarrollo de sistemas de enseñanza-aprendizaje asistidos por computador con el objetivo de crear sistemas más inteligentes.

Ovalle y Jiménez (2006) puntualizan que "algunas de las técnicas de la inteligencia artificial son Planificación Instruccional, Razonamiento Basado en Casos (CBR), ITS, Ambientes Colaborativos de Aprendizaje (CSCL) y Sistemas Multi-agente (MAS), entre otros" (p. 99).

Los maestros y tutores — según Morales (2007)— juegan un papel crucial en el proceso de enseñanza-aprendizaje, pues proporcionan al estudiante retroalimentación sobre lo estudiado y orientación sobre la ruta a seguir, con lo que se logra un cierto grado de personalización del proceso educativo a las necesidades y aptitudes específicas de cada estudiante.

Ovalle y Jiménez (2006) refieren que la investigación en este campo es muy ambiciosa, ya que este tipo de sistemas plantean el desarrollo de herramientas que monitoricen de forma inteligente y analicen el grado de atención y el nivel de productividad de los estudiantes en cualquier área del conocimiento. En la investigación de Rodríguez (2019) se hace referencia al diseño de los ITS, los cuales usan técnicas de la AI, disciplina que estudia la creación y diseño de entidades capaces de razonar por sí mismas (Cataldi y Lage, 2009), la visión por generar formas que imiten el comportamiento de la inteligencia 


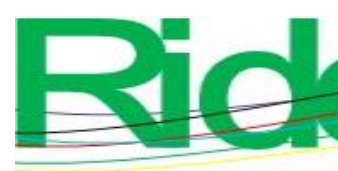

Revista Iberoamericana para la Investigación y el Desarrollo Educativo ISSN $2007-7467$

humana y la creciente demanda del sistema educativo para medir el rendimiento del estudiante. En este contexto, la AI de la mano de las TIC crearon la instrucción asistida por computadora (CAI), sistema de instrucciones computacionales que posee dos características importantes: el estudiante y la computadora, la cual sirve como vehículo para las instrucciones, de ahí que no sea un método de enseñanza (Huapaya, 2009).

Un sistema tutorial inteligente (ITS) se define como sistemas computacionales diseñados para impartir instrucción y apoyar inteligentemente los procesos de enseñanzaaprendizaje mediante la interacción con el alumno (Arias, Jiménez y Ovalle, 2009). Desde esta perspectiva, un ITS es un tipo de ambiente interactivo que está diseñado para el aprendizaje individual y se distingue de los otros tipos por su capacidad para modelar el estado cognitivo del usuario, permitiendo brindar consejos sensibles al contexto y retroalimentar en todos los pasos de un proceso de aprendizaje (Graesser, Chipman, Haynes y Olney, 2005). Su objetivo principal, además de dominar un área de conocimiento en específico, es desarrollar una metodología que se adapte al alumno e interactúe dinámicamente con él (Hernández y Rengifo, 2015).

Se asume que al aprender a programar computadoras, el estudiante desarrolla ciertas habilidades, pero los profesores manifiestan que no todos los alumnos las desarrollan con la misma eficiencia al tener deficiencias en aptitud y actitud (Quiroga, 2016).

La enseñanza de programación dentro de la Universidad Politécnica de Victoria (UPV) toma el grupo de estudiantes como una entidad dinámica, es decir, pasa de un tema tras otro, pero es en ese trayecto donde el estudiante no alcanza el dominio del tema, por lo que carece de las herramientas suficientes para abordar el siguiente. Esto causa que progresivamente el alumno se vea superado por las actividades de la materia y termine por darse por vencido y alejarse de la programación. Por tal motivo, la falta de dominio de los temas al momento de desarrollar software produce un desinterés en los alumnos, lo que representa un problema en los resultados académicos provocando altos índices de reprobación y deserción escolar dentro de la UPV.

En el área de la programación computacional el alumno debe desarrollar habilidades de inteligencia emocional y estrategias de solución de problemas. Las habilidades que puede destacar dentro de un estudiante son:

- Establecer un plan detallado de solución del problema.

- Dominio de un lenguaje de programación.

- Hacer pruebas y depuración de código. 


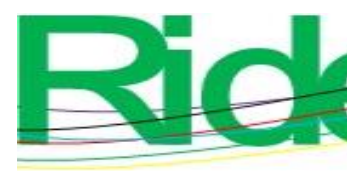

Revista Iberoamericana para la Investigación y el Desarrollo Educativo ISSN 2007 - 7467

De forma específica, las materias de desarrollo de software dentro de la UPV buscan que el estudiante sea capaz de abstraer de la realidad los elementos de un problema que impliquen la automatización de las tareas para el manejo de información, analizar, moldear y describir componentes necesarios para la solución de problemas con la computadora.

Para el apoyo del aprendizaje dentro del aula en temas relacionados con el desarrollo de software, existen los ITS que — a través de la AI - sirven de ayuda para detectar el aprendizaje de los estudiantes de manera oportuna y brindar una alternativa de conocimiento.

Los ITS son sistemas diseñados para replicar la efectividad de la tutoría humana en herramientas digitales. La efectividad de la tutoría individual en la instrucción en grupos grandes se ha establecido con experimentos con tutores humanos. La tutoría es una forma de atención educativa donde el profesor apoya a un estudiante o a un grupo de estudiantes de una manera sistemática por medio de la estructuración de objetivos, programas, organización por áreas, técnicas de enseñanza apropiadas e integración de grupos conforme a ciertos criterios y mecanismos de monitoreo y control, entre otros (Crow, Luxton-Reilly y Wünsche, 2018).

\section{Metodologías para el desarrollo de ITS}

De acuerdo con Rodríguez (2019), existen varias arquitecturas para desarrollar un sistema tutor inteligente, por lo que también son múltiples las metodologías que se emplean con el mismo objetivo; una de ellas es elaborada por Salgueiro, Costa, Cataldi, Lage, GarcíaMartínez (2005), en la cual proponen un nuevo enfoque, sin alejarse de la estructura clásica, que hace énfasis sobre todo en el módulo del tutor. Este se sustenta en la idea de que mientras más conocimiento instruccional se posea mejor explicado será el contenido y las actividades, lo que se traducirá en mayor conocimiento para el alumno.

Cataldi y Lage (2009), a su vez, proponen una metodología enfocada en el módulo del alumno. Los autores indican que un STI que posea un módulo del alumno muy detallado garantiza la inteligencia del sistema, ya que se adapta a las necesidades de los estudiantes.

Rodríguez (2019) explica que existen metodologías que persiguen la integración de un sistema gestor de aprendizaje (SGA) con un STI (Tarongí, 2010), dotando así a estos últimos con un enfoque basado en la Web. En la integración de los componentes del ITS se establecen las relaciones indicando cuál es la equivalencia entre los datos de las distintas tablas de la base de datos. 


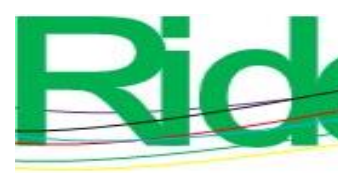

Revista Iberoamericana para la Investigación y el Desarrollo Educativo ISSN 2007 - 7467

La primera relación que se encuentra en el módulo del alumno es el estilo de aprendizaje, que determina el método pedagógico del módulo del tutor. A partir de los distintos tipos de métodos pedagógicos, se han establecido las equivalencias con las diferentes dimensiones de los estilos de aprendizaje. De este modo, se marca el estilo que predomina en cada método, según las características del alumno y según sus carencias; estas se toman en cuenta con el objetivo de instruirlo de la mejor forma posible.

Otras de las relaciones que existen entre el módulo del alumno y el módulo del tutor son la base para definir las actividades que realizará el alumno, las cuales vienen condicionadas por el componente estilo de aprendizaje, y están marcadas por el componente nivel de conocimiento. El componente estilo de aprendizaje condiciona el formato en que se presentarán los objetos, mientras que el nivel de conocimiento marca la complejidad de estos.

Por otra parte, la interfaz viene modelada por el estilo de aprendizaje, aunque esta relación no es muy vinculante, ya que el alumno tiene libertad para modificarla y adaptarla a su gusto; asimismo, se guardan los cambios que realice y se mantienen para las siguientes sesiones de trabajo que establezca.

A modo de inicialización de la aplicación ITS se pueden tomar en consideración las pautas establecidas donde se han analizado las características que presentan los diferentes tipos de herramientas y su relación con las dimensiones del estilo de aprendizaje.

El objetivo principal del sistema de tutoría inteligente es identificar el nivel de aprendizaje sobre el área del desarrollo del software a través de un algoritmo de inteligencia artificial, el cual permitirá detectar de manera temprana el nivel de dominio de los temas relacionados con la programación computacional para ofrecer una alternativa de solución a manera de ejercicios para fortalecer el aprendizaje significativo. El sistema permitirá al estudiante ofrecer soluciones para aprender a desarrollar software y fortalecer así los conocimientos sobre programación computacional.

Existen diferentes librerías de inteligencia artificial que permiten el desarrollo de algoritmos para la creación de sistemas de tutores inteligentes. Por ejemplo, Tensorflow es una biblioteca de código abierto para aprendizaje automático a través de un rango de tareas, desarrollado por Google para satisfacer sus necesidades de sistemas capaces de construir y entrenar redes neuronales para detectar y descifrar patrones y correlaciones, análogos al aprendizaje y al razonamiento usados por los humanos (Tensorflow, 2019). 

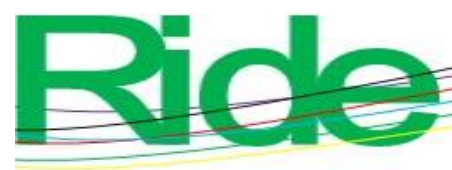

Revista Iberoamericana para la Investigación y el Desarrollo Educativo ISSN 2007 - 7467

El uso de herramientas de inteligencia artificial — como lo es el caso de Tensorflow para la construcción de sistemas de tutoría inteligente- permite generar enseñanzas en tiempo real que favorecen la discusión libre entre las herramientas tecnológicas y el alumno.

En la figura 1 se presenta gráficamente la metodología que se sigue como desarrollo de un ITS basado en la Web; esta consta de 4 etapas:

a) Selección de la plataforma gestor de aprendizaje (SGA).

b) Diseño de módulo del alumno.

c) Diseño de modulo de tutor.

d) Diseño de módulo de dominio.

Figura 1. Metodología para la construcción de un ITS basado en la Web

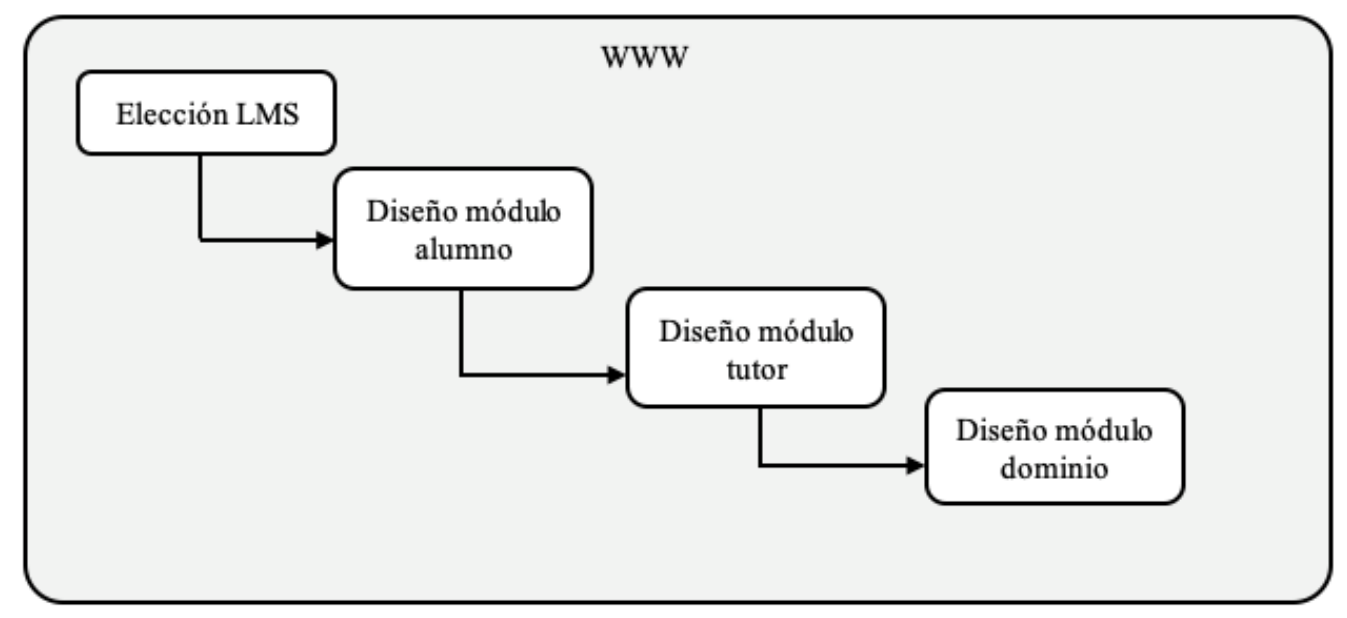

Nota: El diagrama muestra los módulos y la interconexión entre ellos para la construcción de un ITS basado en la Web.

Fuente: Elaboración propia

\section{Selección de la plataforma gestor de aprendizaje (SGA)}

Existen diferentes plataformas SGA que apoyan el proceso de enseñanza-aprendizaje. En el desarrollo de esta investigación, para la construcción de un ITS aplicado en la enseñanza de algoritmos computacionales a nivel licenciatura, se eligió la plataforma Moodle por ser de código abierto y completamente modificable. Es una opción para integrar el ITS por su estructura modular, lo cual ofrece la opción de agregar o eliminar código sin afectar la operación del sistema de gestión del aprendizaje (del inglés learning management system o LMS); asimismo, integra gestores de base de datos diversos, como lo son PostgreSQL y MySQL, que son de fácil instalación. 


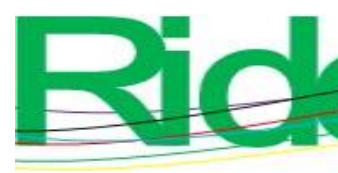

Revista Iberoamericana para la
Investigación y el Desarrollo Educativo
ISSN $2007-7467$

En este apartado se establecen las relaciones entre los principales componentes que forman parte del ITS; la primera relación que se encuentra en el módulo del alumno es el estilo de aprendizaje, lo que determina el método pedagógico del módulo de tutor. A partir de los distintos tipos de métodos pedagógicos, se han establecido las equivalencias con las diferentes dimensiones de los estilos de aprendizaje, se marca el estilo que predomina en cada método, según las características del alumno y según sus carencias; estas se toman en cuenta con el objetivo de instruirlo de la mejor forma posible (Suárez, Arencibia Rodríguez y Pérez Fernández, 2016).

\section{Diseño de módulo del alumno}

El diseño del módulo del alumno sucede cuando se analizan las debilidades y fortalezas del alumno en el momento de asimilar los contenidos de una asignatura. Proporciona información que puede ser retroalimentada a través del ITS y tiene estrecha vinculación con los contenidos y el método pedagógico del módulo del tutor, con los que se instruyen al estudiante, así como con la interfaz de usuario en la que se presentan los contenidos por el módulo de dominio (Suárez et al., 2016).

Para tener una idea del nivel de conocimiento que posee el alumno previo a la interacción con el ITS, se realiza una minería de datos sobre el promedio de las notas de la asignatura Introducción a la Programación Computacional, que toman actualmente en la Universidad Politécnica de Victoria. Mediante una red neuronal de entrenamiento profundo construida con herramientas de inteligencia artificial, se clasifica al alumno, según el conjunto de términos lingüísticos, en competente, básico avanzado, básico y no competente.

\section{Diseño de módulo de tutor}

El módulo del tutor es el motor de ejecución del sistema adaptativo, pues codifica los métodos de enseñanza que son apropiados para el dominio objetivo y el estudiante. Este selecciona la intervención educativa más adecuada en función del conocimiento y estilos de aprendizaje de los alumnos (Suárez et al., 2016).

El diseño del módulo del tutor consta de codificar los métodos de enseñanza que son apropiados para el dominio objetivo de los estudiantes, selecciona la intervención educativa adecuada en función del conocimiento y estilos de aprendizaje de los estudiantes. Las características del alumno determinan el formato en que desean que se les presenten los 


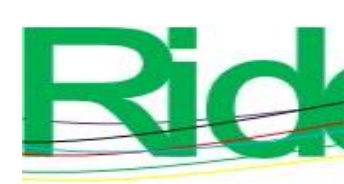

Revista Iberoamericana para la Investigación y el Desarrollo Educativo ISSN 2007 - 7467

materiales junto con la complejidad de los contenidos que debe aprender; esto permite planificar una ruta de aprendizaje a través de una red neuronal para que se ofrezca una propuesta de trabajo en el módulo del alumno.

\section{Diseño de módulo de dominio}

Finalmente, el módulo de dominio consiste en integrar una red neuronal de aprendizaje profundo a través de librerías de inteligencia artificial que permitirá comparar las acciones y elecciones del alumno en un sistema experto con el objetivo de evaluar lo que el usuario conoce y desconoce.

El módulo del dominio está compuesto por la ruta de aprendizaje que viene definida por el módulo del tutor. Esta ruta de aprendizaje contiene la secuenciación a aplicar para definir y estructurar el tema, que se debe materializar en una serie de actividades (tareas, cuestionarios, encuestas, etc.) que vienen diseñadas y condicionadas por el curso y que se ofrecen al alumno a través de una interfaz de usuario, adaptadas a las características del estudiante (Suárez et al., 2016).

La interacción del alumno con las actividades en la interfaz produce una serie de informes que se almacenan en una base de datos para verificar el progreso del alumno. Dicho análisis se obtiene del promedio de las calificaciones obtenidas producto de la interacción del estudiante con el ITS en la plataforma Moodle; con ello, a través de una red neuronal, se logra clasificar el aprendizaje del alumno en un determinado tema.

\section{Metodología}

Con base en la pregunta de investigación ¿cuáles son los usos de los sistemas de tutoría inteligente en la educación?, se presenta una metodología cuantitativa exploratoria para mostrar los resultados del uso de un ITS basado en la Web que ayuda en el aprendizaje de algoritmos computacionales y a identificar las causas que afectan el desempeño académico de los alumnos.

En esta sección se explora el uso de los ITS en la educación superior a través del análisis de la literatura; asimismo, se hace una descripción de la metodología utilizada para su desarrollo y las características de los programas de enseñanza asistidos por computadora que utilizan la IA como base para la representación del conocimiento para dirigir una estrategia de enseñanza preparada para comportarse como un experto. 


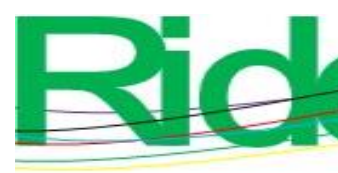

Revista Iberoamericana para la
Investigación y el Desarrollo Educativo
ISSN $2007-7467$

En la primera fase de la metodología se hizo una investigación que se divide en dos vertientes: la investigación de la literatura y la investigación aplicada. La primera implicó el análisis de los ITS utilizados para el apoyo a la docencia y la prueba de teorías para satisfacer intereses intelectuales, mientras que en la investigación aplicada se empleó el conocimiento para la resolución de problemas prácticos.

La revisión de la literatura permitió esquematizar los estudios disponibles en la implementación de los ITS, lo que proporcionó un campo de acción para la realización de nuevas investigaciones que resaltan el progreso de las implementaciones generando cada vez sistemas tutores con alto sentido de inteligencia empleando nuevas técnicas de análisis cognitivos.

Asimismo, se hizo un análisis exploratorio de los diversos ITS implementados en las diferentes ciencias de la educación superior; con base en esto, se presentan los modelos para la construcción del ITS basado en la Web, los cuales darán apoyo en el aprendizaje de algoritmos computacionales dentro de las asignaturas relacionadas con el desarrollo de software y algoritmos computacionales.

Al ser un tema de estudio innovador para la investigación de los diferentes ITS en la educación de nivel superior, no se consideró un rango de fechas específico de publicación de investigaciones, por lo que la totalidad de los hallazgos se concentró en resultados.

Para la selección del conjunto inicial del análisis exploratorio se hizo la consulta del título sistemas de tutoría inteligente en la educación. El conjunto inicial se depuró mediante la lectura completa de cada publicación.

\section{Modelos de sistemas de tutoría inteligente}

Arias et al. (2009) proponen un modelo de planificación instruccional en sistemas tutoriales inteligentes que se basa en el nivel de conocimientos de estudiantes, en la teoría de la planificación de la AI y en la estructura de cursos aplicada en el en el sistema tutorial inteligente (cursos inteligentes adaptativos). Este modelo permite generar planes de actividades adaptados a las características de los estudiantes; además, a medida que los estudiantes avanzan en un curso, van adquiriendo nuevo conocimiento que les permite a su vez habilitar nuevos temas. De esta manera se puede lograr la planificación instruccional basada en el secuenciamiento del currículo (Sánchez, Cabrera y Martínez, 2015). 

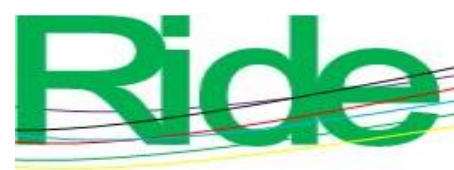

Revista Iberoamericana para la Investigación y el Desarrollo Educativo ISSN 2007 - 7467

En el trabajo de Ovalle y Jiménez (2006) se pone en evidencia un ambiente inteligente distribuido de aprendizaje donde se integran los ITS y los ambientes colaborativos de aprendizaje, se implementan técnicas de IA específicamente en el área del aprendizaje automático, conocido como razonamiento basado en casos, con lo cual se consigue una educación individualizada y colaborativa (Sánchez et al., 2015).

En Cataldi y Lage (2009) se expone una arquitectura básica para los STI, donde se muestra el módulo del tutor. Esta arquitectura presenta una forma más efectiva de comunicación entre el usuario y el sistema, ya que considera el estilo de aprendizaje del estudiante y propone integrar los agentes inteligentes para el desarrollo de los sistemas tutores inteligentes.

En la figura 2 se muestra la arquitectura de un ITS, en la cual se observan cada una de las partes que lo conforman: 1) el módulo de dominio o experto representa el conocimiento; aquí intervienen metodologías de IA como las redes neuronales de clasificación y aprendizaje profundo a través del uso herramientas como Tensorflow, 2) el módulo del estudiante en donde su principal función es capturar el aprendizaje desde el módulo de dominio; 3) el módulo tutorial contiene las estrategias, metodologías e instrucciones que se ajustan a las necesidades del estudiante sin la intervención del ser humano. El objetivo de este elemento es reducir al mínimo la diferencia del conocimiento entre el experto y estudiante (Sánchez et al., 2015). El último módulo del ITS se denomina 4) módulo de entorno, el cual es el gestor de interacción entre todos los componentes del sistema y controla la interfaz de usuario entre la computadora y el ser humano mostrando herramientas de usabilidad y experiencia de usuario para el empleo adecuado del ITS. 

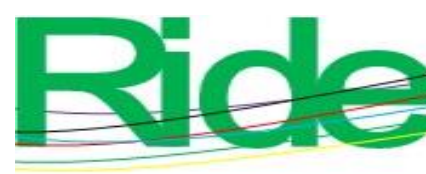

\section{Revista Iberoamericana para la Investigación y el Desarrollo Educativo ISSN 2007 - 7467}

Otro trabajo importante sobre el uso de los ITS es el de Cabada, Barrón y Olivares (2014), los cuales presentan un ITS para la enseñanza-aprendizaje de la matemática, esto con el fin de identificar el estado emocional del estudiante. El software lleva a cabo un análisis emocional a través de una red neuronal artificial, la cual tiene en cuenta el tiempo y los errores dentro de un problema matemático, así como las imágenes de reconocimiento facial del estudiante. En este trabajo se demuestra que el reconocimiento de emociones de los alumnos juega un papel importante en el proceso de aprendizaje.

En la investigación de Oulhaci, Tranvouez, Espinasse y Fournier (2013) se propone un ITS denominado Simfor, el cual mediante la gamificación entrena a no profesionales sobre la gestión de riesgos. El juego consta de módulos de creación de escenarios que simulan un acontecimiento con factor de riesgo para la interacción con el usuario. Es en este proceso formativo donde el usuario adquiere conocimiento sobre el riesgo y cómo debe enfrentarlo.

\section{Resultados}

Una vez revisada la literatura, en la tabla 1 se detallan las publicaciones cronológicamente ordenadas que cumplen los criterios de interés y de exclusión previamente definidos:

Tabla 1. Usabilidad por áreas de especialización de los sistemas de tutoría inteligente

\begin{tabular}{|l|c|c|}
\hline \multicolumn{1}{|c|}{ Autores } & Nombre & Área \\
\hline Rodríguez et al. (2013) & Malvi & Matemáticas \\
\hline Mitrovic et al. (2013) & SQL-tutor & Base de datos \\
\hline $\begin{array}{l}\text { Arevalillo-Herráez } \text { et } \text { al. } \\
(2013)\end{array}$ & Traductor verbal & Álgebra \\
\hline Cabada et al. (2014) & Aprendizaje matemático & Matemáticas \\
\hline Oulhaci et al. (2013) & Simfor & $\begin{array}{c}\text { Factor de } \\
\text { riesgos }\end{array}$ \\
\hline
\end{tabular}

Fuente: Elaboración propia

En las diferentes áreas de conocimiento que se han implementado ITS, se puede observar el enfoque hacia las ciencias matemáticas y computacionales, lo cual ayuda a identificar sus características y servir de base en la construcción de un nuevo modelo de ITS para el apoyo en el aprendizaje de algoritmos computaciones. 

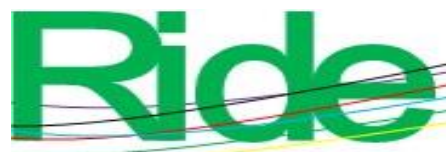

Revista Iberoamericana para la Investigación y el Desarrollo Educativo

ISSN $2007-7467$

En la construcción de un nuevo modelo de ITS como apoyo en el proceso de enseñanza de la codificación de programas computacionales a través de algoritmos, se deben analizar las diferentes técnicas que se han implementado en su desarrollo para generar un nuevo paradigma a través de una estrategia que haga uso de técnicas de la IA que soportarán la construcción de un nuevo ITS.

Por ello, en la tabla 2 se muestra el trabajo de investigación de Sánchez et al. (2015), donde se analizan las técnicas y tendencias de la IA utilizadas como tecnología en el desarrollo de ITS a partir de las investigaciones hechas sobre el uso y desarrollo de tutores inteligentes. 


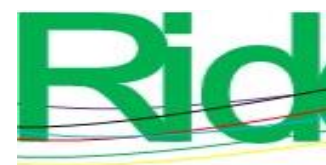

Revista Iberoamericana para la Investigación y el Desarrollo Educativo ISSN 2007 - 7467

Tabla 2. Técnicas de inteligencia artificial implementadas por los respectivos autores

\begin{tabular}{|c|c|}
\hline Técnicas de IA & Autores que la utilizan \\
\hline Lógica difusa & Peña, C., Marzo, J., De la Rosa, J., Fabregat, R. \\
\hline $\begin{array}{l}\text { Razonamiento basado en } \\
\text { casos }\end{array}$ & $\begin{array}{l}\text { Peña, C., Marzo, J., De la Rosa, J., Fabregat, R; Ovalle, } \\
\text { D. y Jiménez, J. }\end{array}$ \\
\hline Agentes inteligentes & $\begin{array}{l}\text { Cataldi, Z., Salgueiro, F., Costa, G., Calvo, P., Méndez, } \\
\text { P., Rendón, J., Lage, F. }\end{array}$ \\
\hline Red neuronal artificial & $\begin{array}{l}\text { Salgueiro, F., Cataldi, Z., Lage, F., García-Martínez, R; } \\
\text { Arnau, D. Arevalillo-Herráez, M., Puig, L., González- } \\
\text { Calero, J.; Sánchez, R., Bartel, Ch., Brown, E., DeRosier, } \\
\text { M.; Oulhaci, M. A., Tranvouez, E., Espinasse, B., } \\
\text { Fournier, S.; Zhiping, L., Tianwei, X., Yu, S; } \\
\text { Cabada, R. Barrón, M. y Olivares, J. M. J. }\end{array}$ \\
\hline Redes bayesianas & Cataldi, Z., Salgueiro, F., Lage, F., García-Martínez, R. \\
\hline Lingüística difusa & $\begin{array}{l}\text { Badaracco, M., Martínez, L; Azoulay-Schwartz, R., Hani, } \\
\text { Z. }\end{array}$ \\
\hline $\begin{array}{l}\text { Representación de } \\
\text { conocimiento }\end{array}$ & Arevalillo-Herráez, M., Arnau, D., Marco-Giménez, L. \\
\hline Lingüística computacional & Xuechen, He; Ferreira, A., Kotz, G. \\
\hline $\begin{array}{l}\text { Procesamiento del lenguaje } \\
\text { natural }\end{array}$ & $\begin{array}{l}\text { Latham, A., Crockett, K., McLean, D.; Gorrostieta, J., } \\
\text { González, S., López, A.; Jackson, T., Graesser, A. }\end{array}$ \\
\hline Visión artificial & $\begin{array}{l}\text { Sathyanarayana, S., Littlewort, G., Bartlett, M.; Qui-rong, } \\
\text { Chen. }\end{array}$ \\
\hline
\end{tabular}

Fuente: Elaboración propia con base en Sánchez et al. (2015)

\section{Discusión}

Analizando la literatura sobre los diferentes tipos de ITS existentes en diversas disciplinas, se observa que para su desarrollo los autores utilizan técnicas de IA implementando redes neuronales y teorías de la educación, como lo son la psicología cognitiva. En tal sentido, Mitrovic y Ohlsson (1999) muestran en su sistema un modelo del estudiante que determina acciones pedagógicas; asimismo, Arevalillo-Herráez et al. (2013) describen un sistema tutorial inteligente que se centra en la etapa de traducción de problemas 


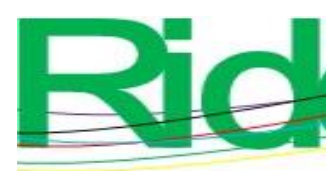

Revista Iberoamericana para la
Investigación y el Desarrollo Educativo
ISSN $2007-7467$

verbales en notación simbólica, lo que permite aprender a solucionar problemas algebraicos de manera verbal. Por otro lado, se analiza el trabajo de Cabada et al. (2014), en donde desarrollan un ITS para aprender matemáticas tomando como variable sobresaliente el estado emocional del estudiante; para este trabajo hacen uso de una red neuronal artificial que toma en cuenta el tiempo de solución de problemas y el número de errores que se presenta al momento resolver problemas matemático. Finalmente, se analiza el ITS Simfor, sistema desarrollado por Oulhaci et al. (2013) mediante el cual, a través de la gamificación con los estudiantes, se entrena sobre la identificación y gestión de riesgos físicos.

Como trabajo futuro - a partir de los hallazgos de la literatura sobre los ITS desarrollados y las metodologías implementadas - se toman las bases para la construcción de un ITS para apoyar la enseñanza del área de los algoritmos computacionales. Para esto, se considera fundamental la implementación de un ITS para el desarrollo del razonamiento, pensamiento analítico y lógico en los estudiantes de la carrera de ingeniería en Tecnologías de la Información de la Universidad Politécnica de Victoria (UPV), ya que las materias relacionadas con el desarrollo de software son las que tienen un alto índice de reprobación. La tabla 3 muestra el comportamiento de reprobación de materias por cuatrimestre dentro del periodo enero 2019-agosto 2020. Es importante señalar que las materias identificadas son las que tienen relación en el desarrollo de software y que a su vez se han impartido una o más ocasiones dentro del periodo señalado. 
Tabla 3. Comportamiento de reprobación de materias por cuatrimestre: periodo enero 2019-agosto 2020 del programa académico (PE) de ITI de la UPV

\begin{tabular}{|c|c|c|c|c|c|}
\hline Materia & $\begin{array}{c}\text { Enero- } \\
\text { abril } \\
2019\end{array}$ & $\begin{array}{c}\text { Mayo- } \\
\text { agosto } \\
2019\end{array}$ & $\begin{array}{l}\text { Septiembre } \\
\text {-diciembre } \\
2019\end{array}$ & $\begin{array}{c}\text { Enero- } \\
\text { abril } \\
2020\end{array}$ & $\begin{array}{c}\text { Mayo- } \\
\text { agosto } \\
2020\end{array}$ \\
\hline $\begin{array}{l}\text { Programación orientada a } \\
\text { objetos }\end{array}$ & & & & & $83 \%$ \\
\hline $\begin{array}{l}\text { Graficación por } \\
\text { computadora avanzada }\end{array}$ & & & & $71 \%$ & \\
\hline $\begin{array}{l}\text { Fundamentos de } \\
\text { programación orientada a } \\
\text { objetos }\end{array}$ & & & & $67 \%$ & \\
\hline Minería de datos aplicada & $62 \%$ & & & & \\
\hline $\begin{array}{l}\text { Cómputo en dispositivos } \\
\text { móviles }\end{array}$ & & $44 \%$ & & & $43 \%$ \\
\hline $\begin{array}{l}\text { Introducción a la } \\
\text { graficación por } \\
\text { computadora }\end{array}$ & $25 \%$ & & $59 \%$ & $35 \%$ & \\
\hline Estructura de datos & $42 \%$ & $27 \%$ & $39 \%$ & & \\
\hline $\begin{array}{l}\text { Tecnología y aplicaciones } \\
\text { web }\end{array}$ & & $36 \%$ & $49 \%$ & & $32 \%$ \\
\hline $\begin{array}{l}\text { Matemáticas básicas para } \\
\text { computación }\end{array}$ & $33 \%$ & $34 \%$ & & $44 \%$ & \\
\hline Probabilidad y estadística & & $34 \%$ & $24 \%$ & & \\
\hline $\begin{array}{l}\text { Introducción a la } \\
\text { programación }\end{array}$ & & & $36 \%$ & $21 \%$ & \\
\hline Negocios electrónicos & $39 \%$ & & & $20 \%$ & $32 \%$ \\
\hline $\begin{array}{l}\text { Introducción a las } \\
\text { tecnologías de información }\end{array}$ & $29 \%$ & & & & \\
\hline Programación & & $32 \%$ & $24 \%$ & & \\
\hline Base de datos & & & & & $28 \%$ \\
\hline Programación web & $27 \%$ & $28 \%$ & & & \\
\hline $\begin{array}{l}\text { Integración de tecnologías } \\
\text { de la información }\end{array}$ & & $25 \%$ & & & \\
\hline $\begin{array}{l}\text { Introducción a las bases de } \\
\text { datos }\end{array}$ & $20 \%$ & & & & \\
\hline
\end{tabular}

Nota: Esta tabla representa los resultados de reprobación de las materias relacionadas al desarrollo de software en el periodo comprendido entre enero 2019 y agosto 2020. En ella se aprecian los cuatrimestres donde las asignaturas se impartieron y el nivel de reprobación que arrojaron los resultados en cada uno de ellos, según el Sistema Integral de Información de la UPV (SIIUPV 2020). 


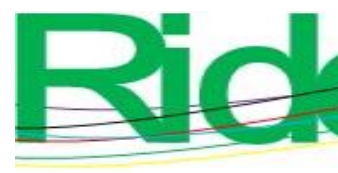

Revista Iberoamericana para la Investigación y el Desarrollo Educativo ISSN $2007-7467$

Se elige la implementación de esta herramienta, ya que se considera eficiente en el proceso de enseñanza-aprendizaje. Además, ha demostrado en la literatura tener en cuenta las capacidades cognitivas de cada estudiante y adaptarse a sus capacidades de aprendizaje.

\section{Conclusiones}

Con base en la exploración de la literatura sobre el uso de los ITS en la educación superior y asumiendo que el objetivo principal de los ITS es la identificación del nivel de aprendizaje sobre un área de la ciencia para ofrecer un soporte en el proceso de enseñanzaaprendizaje para la estimulación de los estudiantes, algunos sistemas hacen uso de la gamificación para no generar desinterés y aburrimiento. Por eso, con base en el análisis exploratorio de los ITS utilizados en la educación a nivel superior como apoyo en el aprendizaje en diferentes áreas de la ciencia, se observa que la incorporación de los ITS en la educación ayudan a resolver la problemática de la enseñanza de considerar que todos los estudiantes tienen las mismas necesidades de aprendizaje y la misma forma de aprender. En relación con esto, los ITS juegan un papel muy importante, puesto que han sido creados para identificar las debilidades y necesidades de aprendizaje individuales y así establecer una metodología de enseñanza apropiada según cada caso.

El objetivo de los ITS no es el reemplazo del tutor humano, sino reforzar la enseñanza dentro y fuera del aula. En la implementación de los ITS el tutor humano eficientiza sus actividades haciéndose cargo en forma personalizada de las tareas que el ITS no puede realizar. Bajo este panorama de la enseñanza se considera al alumno como el centro principal en el proceso educativo, pues es él quien regula sus aprendizajes. De esta forma, se logra crear una visión de enseñanza donde es el estudiante el protagonista del modelo y sus necesidades la prioridad.

Como trabajo futuro se consideran los hallazgos encontrados en la literatura para servir de base en la construcción e implementación de un ITS en la carrera de ITI de la UPV para mejorar el rendimiento académico de los alumnos de las áreas del desarrollo de software. 


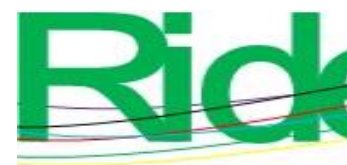

\section{Revista Iberoamericana para la Investigación y el Desarrollo Educativo ISSN $2007-7467$}

Cabada, R., Barrón, M. y Olivares, J. M. (2014). Reconocimiento automático y aspectos éticos de emociones para aplicaciones educativas. Komputer Sapiens, 27-33.

Carbonell, J. R. (1970). AI in CAI: An artificial intelligence approach to computer assisted instruction. IEEE transaction on Man Machine System, 11(4), 190-202.

Cataldi, Z. y Lage, F. (2009). Sistemas tutores inteligentes orientados a la enseñanza para la $\begin{array}{llll}\text { comprensión. } & \text { Edutec, } & \text { (28). }\end{array}$ http://www.edutec.es/revista/index.php/edutec-e/article/view/456

Clancey, W. J. (1991). Intelligent tutoring systems: A tutorial survey, en App. Artificial Intelligence: A Sourcebook. McGraw-Hill.

Crow, T., Luxton-Reilly, A. and Wünsche, B. (2018). Intelligent tutoring systems for programming education: a systematic review. 20th Australasian Computing Education Conference, 53-62. Doi: https://doi.org/10.1145/3160489.3160492

Galvin, T. (1994). Mebuilder: An Object-Oriented Lesson Authoring System for Procedural Skills (9/94) (tesis doctoral). Retrieved from https://calhoun.nps.edu/handle/10945/30938 Gertner, A. S., Conati, C. and VanLehn, K. (1998). Learning Procedural help in Andes: Generating hints using a Bayesian network student model. Research \& Development. American Association for Artificial Intelligence. Retrieved from https://asu.pure.elsevier.com/en/publications/procedural-help-in-andes-generatinghints-using-a-bayesian-networ

Gertner, A.S. y VanLehn, K. (2000). Andes: A Coached Problem Solving Environment for Physics. Lecture Notes In Computer Science; Vol. 1839 Proceedings of the 5th International Conference on Intelligent Tutoring Systems. Pages: 133 - 142

Giraffa, L., Nunes, M. A. and Viccari, R. M. (1997). Multi-Ecological: A Learning Environment using Multi-Agent architecture. MASTA'97: Multi-Agent System: Theory and Applications. Proceedings. Coimbra: DE-Universidade de Coimbra.

Graesser, A. C., Chipman, P., Haynes, B. and Olney, A. (2005). AutoTutor: an intelligent tutoring system with mixed - iniciative dialogue. IEEE Transactions on Education, 48(4). Retrieved from https://ieeexplore.ieee.org/stamp/stamp.jsp?tp=\&arnumber=1532370

Guzmán, E., y Conejo, R. (2005). Un modelo de evaluación cognitiva basado en Tests Adaptativos Informatizados para el diagnóstico en Sistemas Tutores Inteligentes. (tesis de pregrado). Universidad de Málaga. Recuperado de: http://www.lcc.uma.es/repository/fileDownloader?rfname=LCC1406.pdf 


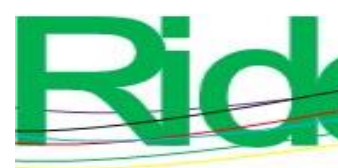

Revista Iberoamericana para la Investigación y el Desarrollo Educativo ISSN 2007 - 7467

Hernández, J. y Rengifo, Y. (2015) Los sistemas tutores inteligentes y su aplicabilidad en la educación. Horizontes Pedagógicos, 17(2), 104-115. Recuperado de https://revistas.iberoamericana.edu.co/index.php/rhpedagogicos/article/view/17209

Huapaya, C. (2009). Sistemas tutoriales inteligentes. Un análisis crítico. Facultad de informática. Universidad Nacional de la Plata. Recuperado de https://postgrado.info.unlp.edu.ar/wp-content/uploads/2014/07/Huapaya.pdf

Johnson, W. L. (1986). Intention-based diagnosis of novice programming errors. MorganKauffman.

Mitrovic, A. and Ohlsson, S. (1999). Evaluation of a constraintbased tutor for a database language. International Journal of Artificial Intelligence in Education, 10(3-4), 238-256.

Mitrovic, A., Olhsson, S. and Barrow, D. (2013). The effect of positive feedback in a constraint-based intelligent tutoring system. Computers \& Education, 60(1).

Morales, R. (2007). Modelado del estudiante para ambientes virtuales de aprendizaje en Web. Apertura: Revista de Innovación Educativa, 7(7), 21-35. Recuperado de http://search.ebscohost.com/login.aspx ?direct=true\&db=zbh\&AN=28627025\&site=eho st-live

Oulhaci, M. A., Tranvouez, E., Espinasse, B. and Fournier, S. (2013). Intelligent Tutoring Systems and Serious Game for Crisis Management: a Multi- Agents Integration Architecture. 2013 Workshops on Enabling Technologies: Infrastructure for Collaborative Enterprises.

Ovalle, D. y Jiménez, J. (2006). Ambiente inteligente distribuido de aprendizaje: integración de ITS y CSCL por medio de agentes pedagógicos. Revista EIA, (6), 89-104.

Quiroga, L. (2016). Prototipo de tutor inteligente para el aprendizaje de la programación de computadores (tesis de pregrado). Universidad Católica de Colombia, Bogotá, Colombia. Recuperado de https://repository.ucatolica.edu.co/bitstream/10983/14968/1/PROTOTIPO\%20DE\%20 UN\%20SISTEMA\%20TUTOR\%20INTELIGENTE.pdf

Quispe, R. (2014) Tutor inteligente para fortalecer el aprendizaje de la estructura morfosintáctica a nivel primero de primaria (Tesis de pregrado). Universidad Mayor de San Andrés, La Paz, Bolivia.

Rodríguez, A., Castillo, J. y Lira, A. (2013). Diseño de un sistema tutorial inteligente. Apertura, 5(1), 36-47. 

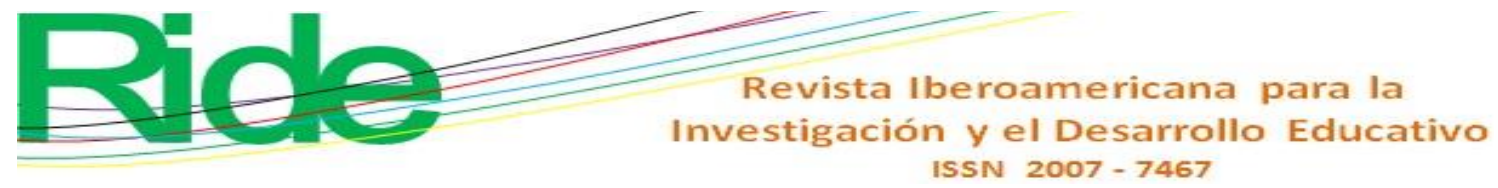

Rodríguez, M. (2019). Sistema de tutoría inteligente aplicado a la enseñanza de programación de computadoras a nivel licenciatura. En Borrego Gómez, D., Cantú Cervantes, D. y Molina Montalvo, H. Educación y tecnologías (pp. 179-189). Editorial Palibrio. Recuperado de https://www.amazon.com.mx/Educaci\%C3\%B3nTecnolog\%C3\%ADas-Daniel-Desiderio-Borrego/dp/1506530214

Rowe N. C. y T. P. Galvin, (1998) "An authoring system for intelligent procedural-skill tutors," en IEEE Intelligent Systems and their Applications, vol. 13, no. 3, pp. 61-69, May-Jun 1998, doi: 10.1109/5254.683211.

Salgueiro, F., Costa, G., Cataldi, Z., Lage,F. y García-Martínez, R. (2005). Nuevo enfoque metodológico para el diseño de los sistemas tutores inteligentes a partir de un acercamiento distribuido. Revista de Informática Educativa y Medios Audiovisuales, 2(4), 25-32. Recuperado de http://laboratorios.fi.uba.ar/lie/Revista/Articulos/020205/A3ago2005.pdf

Sánchez, I. I., Cabrera, J. M. y Martínez, J. E. (2015). Ayudas virtuales como apoyo al aprendizaje inclusivo en la ingeniería. Revista Horizontes Pedagógicos, 17(2), 104-116.

Stevens, A. and Collins, A. (1977). The goal structure of a Socratic tutor. Proc.s of the National ACM Conference. New York: ACM.

Suárez, J. J., Arencibia Rodríguez, Y. y Pérez Fernández, A. C. (2016). Metodología para desarrollar un sistema tutor inteligente basado en la Web para estudiantes de ingeniería. Universidad y Sociedad, 8(4), 108-115.

Tarongí, V. A. (2010). Sistema tutor inteligente adaptativo para laboratorios virtuales y remotos (tesis de master). Valencia: Universidad Politécnica de Valencia.

Tensorflow Core https://www.tensorflow.org/ Consultado el 24-07-2019.

Urretavizcaya, M. (2001). Sistemas inteligentes en el ámbito de la educación. Inteligencia Artificial. Revista Iberoamericana de Inteligencia Artificial, 5(2), 5-12.

VanLehn, K (1988). Student Modelling. M. Polson. Foundations of Intelligent Tutoring systems. Hillsdale. N.J. Lawrence Erlbaum Associates, 55-78.

Wolf, B. (1984). Context Dependent Planning in a Machine (Ph. D. dissertation). University of Massachusetts, Amherst, Massachusetts. 